University of Wollongong

Research Online

Faculty of Engineering - Papers (Archive)

Faculty of Engineering and Information

Sciences

September 2006

\title{
Chandra Observations of Nuclear Outflows in the Elliptical Galaxy NGC 4552 in the Virgo Cluster
}

M. Machacek

Harvard-Smithsonian Center for Astrophysics, Cambridge, USA

P. Nulsen

University of Wollongong

C. Jones

Harvard-Smithsonian Center for Astrophysics, Cambridge, USA

W. R. Forman

Harvard-Smithsonian Center for Astrophysics, Cambridge, USA

Follow this and additional works at: https://ro.uow.edu.au/engpapers

Part of the Engineering Commons

https://ro.uow.edu.au/engpapers/290

\section{Recommended Citation}

Machacek, M.; Nulsen, P.; Jones, C.; and Forman, W. R.: Chandra Observations of Nuclear Outflows in the Elliptical Galaxy NGC 4552 in the Virgo Cluster 2006.

https://ro.uow.edu.au/engpapers/290

Research Online is the open access institutional repository for the University of Wollongong. For further information contact the UOW Library: research-pubs@uow.edu.au 


\title{
CHANDRA OBSERVATIONS OF NUCLEAR OUTFLOWS IN THE ELLIPTICAL GALAXY NGC 4552 IN THE VIRGO CLUSTER
}

\author{
M. Machacek, P. E. J. Nulsen, ${ }^{1}$ C. Jones, and W. R. Forman \\ Harvard-Smithsonian Center for Astrophysics, 60 Garden Street, Cambridge, MA 02138; mmachacek@cfa.harvard.edu \\ Received 2006 January 23; accepted 2006 May 18
}

\begin{abstract}
We use a 54.4 ks Chandra observation to study nuclear outflow activity in NGC 4552 (M89), an elliptical galaxy in the Virgo Cluster. Chandra images in the $0.5-2 \mathrm{keV}$ band show two ringlike features $\sim 1.7 \mathrm{kpc}$ in diameter in the core of NGC 4552, as reported previously by Filho et al. We use spherically symmetric point explosion shock models to argue that the shape of the surface brightness profile across the rims of the rings and the temperature of hot gas in the rings are consistent with a Mach 1.7 shock carrying mean mechanical power $L_{\text {shock }} \sim 3 \times 10^{41} \mathrm{ergs} \mathrm{s}^{-1}$ produced by a $\sim 1.4 \times 10^{55} \mathrm{ergs}$ nuclear outburst $\sim 1-2 \mathrm{Myr}$ ago. We find the gas temperature in the central $\sim 100 \mathrm{pc}$ of the galaxy to be $1.0 \pm 0.2 \mathrm{keV}$, hotter than elsewhere in the galaxy, suggesting that we may be directly observing the reheating of the galaxy ISM by the outburst.
\end{abstract}

Subject headings: galaxies: clusters: general — galaxies: individual (NGC 4552, M89) — intergalactic medium — X-rays: galaxies

Online material: color figure

\section{INTRODUCTION}

X-ray observations of nearby galaxies provide a unique opportunity to directly study the interaction of the central active galactic nucleus (AGN) with the galaxy's surrounding hot gaseous atmosphere. Chandra's high angular resolution permits the identification of outflow shocks, cavities, and buoyant bubbles that directly trace the flow of matter, energy, and momentum away from the nucleus and into the surrounding environment (see, e.g., Young et al. [2002] and Forman et al. [2005] for M87 in the Virgo Cluster, Fabian et al. [2003] for NGC 1275 in the Perseus Cluster, and McNamara et al. [2000] and Nulsen et al. [2005b] for Hydra A).

It is now widely recognized that understanding the nature of these AGN outflows is fundamental to further advances in the understanding of the evolution of structure (galaxies, groups, and clusters) and their hot gas environments, the interstellar medium (ISM) and intracluster medium (ICM). Energy transfer to the ICM through episodic nuclear outbursts from a central supermassive black hole (Binney \& Tabor 1995; Owen et al. 2000) is likely necessary to resolve the order of magnitude discrepancy between the small amount of cool gas measured in the cores of rich clusters (e.g., Tamura et al. 2001; Peterson et al. 2003 and references therein) and that predicted by standard cooling-flow models (Fabian 1994). Simulations demonstrated that the physical mechanisms responsible for the transfer of energy from the resulting relativistic plasma jets, shocks, buoyant bubbles, and sound waves to the surrounding gas are complex and strongly dependent on the age of the outburst (e.g., Heinz et al. 1998; Churazov et al. 2001; Quilis et al. 2001; Brüggen \& Kaiser 2002; Reynolds et al. 2001, 2005; Ruszkowski et al. 2004; Fabian et al. 2005; Heinz \& Churazov 2005; Omma et al. 2004). Young flows are characterized by shocks driven into the ambient medium by the outburst that heat the gas locally (e.g., Heinz et al. 1998; Omma et al. 2004 and references therein). After the outflow matures, fluctuations in the gas ("ripples") passing over buoyant

\footnotetext{
${ }^{1}$ On leave from the University of Wollongong.
}

bubbles may induce Richtmyer-Meshkov instabilities that might dominate the transfer of bubble enthalpy to the ambient gas (Heinz \& Churazov 2005).

Recent studies indicate that feedback from a central AGN may be fundamental to our understanding of galaxy evolution in general. Simulations using the $\Lambda \mathrm{CDM}$ cosmology suggest that nongravitational heating of the ISM by outbursts from the central, supermassive black hole in galaxies is needed to correctly reproduce the observed shape of the galaxy luminosity function and massive galaxy colors at low redshift (Granato et al. 2004; Croton et al. 2006 and references therein). Best et al. (2006) used FIRST and NVSS data to measure the fraction of galaxies that are radioactive above a given $1.4 \mathrm{GHz}$ radio luminosity as a function of that luminosity and central black hole mass. They interpreted their result as a probabilistic estimate of the time a galaxy, with given black hole mass, is active above a given radio brightness, and they combined this result with the Birzan et al. (2004) correlation between the current central radio luminosity and the mechanical luminosity of outbursts to estimate the total energy available from AGNs to heat the ambient ISM. Best et al. (2006) argued that these data showed that heating from AGNs could balance cooling in typical elliptical galaxies, and that most of the AGN heating in these galaxies occurs when the radio luminosity of the central AGN is low, $L_{1.4 \mathrm{GHz}}<10^{31} \mathrm{ergs} \mathrm{s}^{-1}$ $\mathrm{Hz}^{-1}$. Thus, outflows may be important in systems with low- as well as high-luminosity AGNs.

In this work we use a $54.4 \mathrm{ks}$ Chandra observation to study nuclear outburst activity in one such galaxy, NGC $4552(\alpha=$ $12^{\mathrm{h}} 35^{\mathrm{m}} 39^{\mathrm{s}} 8, \delta=12^{\circ} 33^{\prime} 23^{\prime \prime}[\mathrm{J} 2000.0]$ ), an elliptical galaxy located $72^{\prime}$ east of M87 in subcluster A of the Virgo Cluster. In a companion paper (Machacek et al. 2006, hereafter Paper I) we use the same Chandra observation to measure the properties of hot gas in the outer regions of NGC 4552, showing that these properties are consistent with that expected for gas being rampressure-stripped due to NGC 4552's supersonic motion through the Virgo ICM. NGC 4552 is a member of the large sample of nearby elliptical galaxies used to establish correlations between $\mathrm{X}$-ray emission and other ISM tracers so that its global X-ray 

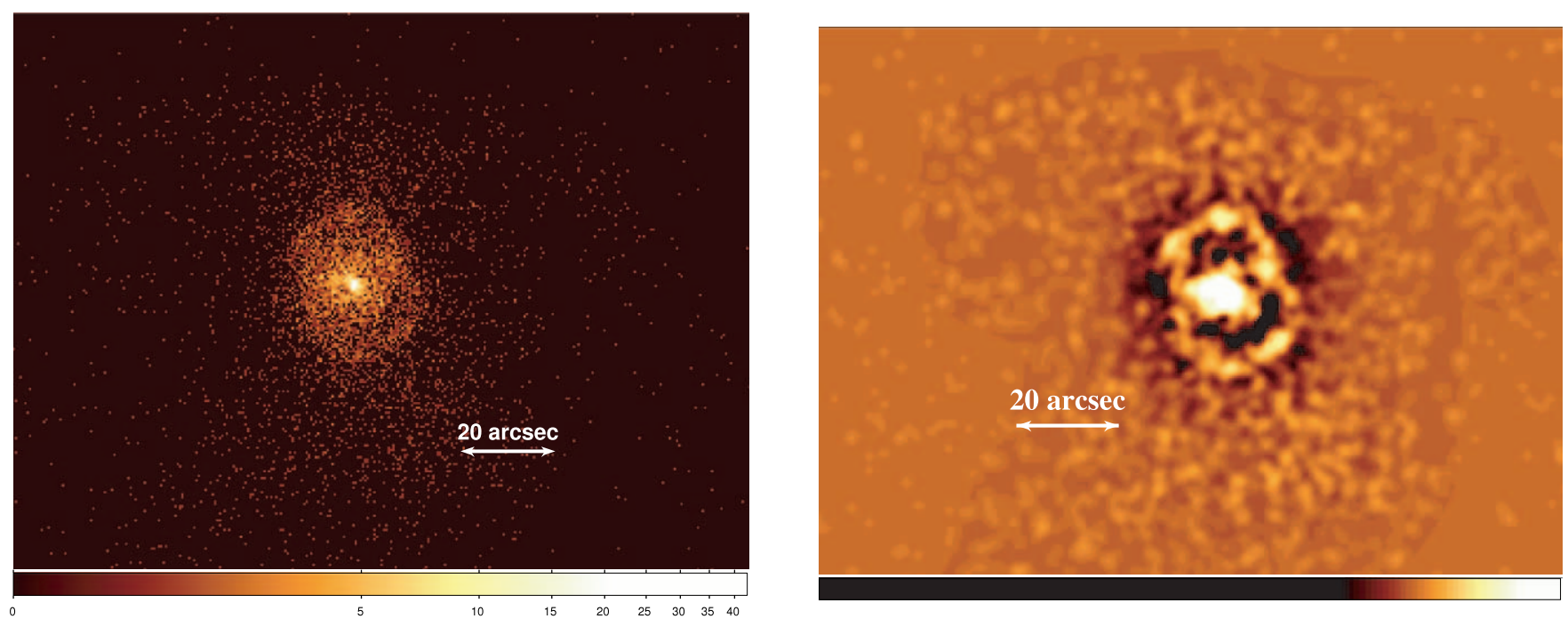

Fig. 1. - Left: Chandra image of the 0.7-1 keV diffuse emission from the inner rings in the central region of NGC 4552.1 pixel $=0$ " $49 \times 0$ " 49 . Right: Unsharp masked image of the same region, where we subtract the $0.7-1 \mathrm{keV}$ diffuse image smoothed with a 5"4 Gaussian kernel from the same image smoothed with a 1" Gaussian kernel to highlight the rings.

properties are well studied (for a brief review, see Paper I and references therein).

Studies of the nuclear properties of NGC 4552 across a wide range of wavelengths strongly suggest the presence of a weak AGN at its center. From optical line ratios, Ho et al. (1997) classify NGC 4552 as a LINER/H II transition object, possessing spectral properties of a low-luminosity AGN contaminated by nearby starforming $\mathrm{H}$ II regions. However, the classification was highly uncertain due to the weakness of the emission lines. HST spectra of the nuclear region by Cappellari et al. (1999) showed a faint broad emission component, suggesting the presence of an AGN and favoring a LINER classification for NGC 4552's nucleus. Since the broad emission-line component was observed in both the allowed and forbidden transitions, the nucleus was considered borderline between LINER types 1 and 2 . Short-term variability of the nucleus is a distinguishing feature of AGN activity. UV variability has been reported for NGC 4552 on timescales of several months (Maoz et al. 2005) to years (Renzini et al. 1995; Cappellari et al. 1999). Although not precluding a compact circumnuclear starburst since variability in the UV on timescales $\lesssim 1$ year is not expected from young stars, the variable flux provides a lower limit on the AGN contribution in this wave band (Maoz et al. 2005).

Radio continuum measurements taken with the Very Large Array (VLA) at $8.4 \mathrm{GHz}$ (Filho et al. 2000) and with the Very Long Baseline Array (VLBA) at $5 \mathrm{GHz}$ (Nagar et al. 2002) show $\mathrm{a} \sim 100 \mathrm{mJy}$ core radio source, unresolved on milliarcsecond scales, at the galaxy center. Variability at the $\sim 20 \%$ level is observed in the 15 and $8.4 \mathrm{GHz}$ radio flux on $\sim$ year timescales (Nagar et al. 2002). Properties of the radio spectrum indicate a nonthermal origin for this radio emission, most likely associated with accretion onto a central supermassive black hole. The measured brightness temperature, $T_{B} \sim 2 \times 10^{9} \mathrm{~K}$ at $5 \mathrm{GHz}$ (Nagar et al. 2002), is too high for this emission to be due to a nuclear starburst $\left(T_{B} \lesssim 10^{4}-10^{5} \mathrm{~K}\right)$ or collection of supernova remnants $\left(T_{B} \lesssim 10^{7} \mathrm{~K}\right)$. Furthermore, the shape of the radio emission spectrum is too flat $(\alpha \sim 0)$ to be associated with a collection of supernova remnants, whose spectral indices are typically $\sim 0.4-0.7$. Filho et al. (2004) attribute the flatness of the observed radio spectrum to ADAF-like accretion onto a central black hole combined with synchrotron emission from parsec-scale jets or outflows. This is supported by the $5 \mathrm{GHz}$ observation of two $\sim 5$ mas diameter, extended features to the east and west of the core, suggestive of parsec-scale jets (Nagar et al. 2002). From the correlation between the black hole mass and central velocity dispersion (Ferrarese \& Merritt 2000; Gebhardt et al. 2000), the mass of the central black hole in NGC 4552 is inferred to be $4 \times 10^{8} M_{\odot}$. In recent Chandra X-ray observations, Filho et al. (2004) identified a hard X-ray source, whose X-ray spectrum is well represented by a $\Gamma=1.51$ power-law component, plus a $k T=0.95 \mathrm{keV}$ Raymond-Smith thermal component with $0.5-2$ and $2-10 \mathrm{keV}$ luminosities of $\log L_{\mathrm{X}}=39.2$ and $39.4 \mathrm{ergs} \mathrm{s}^{-1}$, respectively, that is coincident with the core radio source in NGC 4552. This is also consistent with the presence of a weak AGN.

Our discussion is organized as follows: In $\oint 2$ we briefly review the observations and our data reduction and processing procedures. In $\S 3$ we present the background-subtracted, exposurecorrected image of the central region of NGC 4552 showing two bright ringlike features within $1.3 \mathrm{kpc}$ of the galaxy center. We model the spectral properties of these rings in the central $1.3 \mathrm{kpc}$ of the galaxy, and we use the X-ray surface brightness profile to estimate the density jump across the rim of the rings. We show that these rings are consistent with shocked gas driven outward by recent nuclear activity. In $\S 4$ the spectral properties of the nuclear region are briefly discussed for comparison with earlier work. In $\S 5$ we summarize our results. Unless otherwise indicated, all errors correspond to $90 \%$ confidence levels, and coordinates are $\mathrm{J} 2000$.0. Taking the distance to the dominant elliptical M87 as representative of the distance to subcluster A of the Virgo Cluster containing NGC 4552, the luminosity distance to the cluster is $16.1 \pm 1.1 \mathrm{Mpc}$ (Tonry et al. 2001), and 1" corresponds to a distance scale of $77 \mathrm{pc}$.

\section{OBSERVATIONS AND DATA REDUCTION}

We used a 54.4 ks observation of the Virgo elliptical galaxy NGC 4552 taken with Chandra on 2001 April 22 using the Advanced CCD Imaging Spectrometer array (ACIS; Garmire et al. 1992; Bautz et al. 1998) with ACIS-S (chip S3) at the aimpoint. A detailed discussion of the observation and data analysis are presented in Paper I. We briefly review our procedures here. The data were analyzed using the standard X-ray processing packages, 
TABLE 1

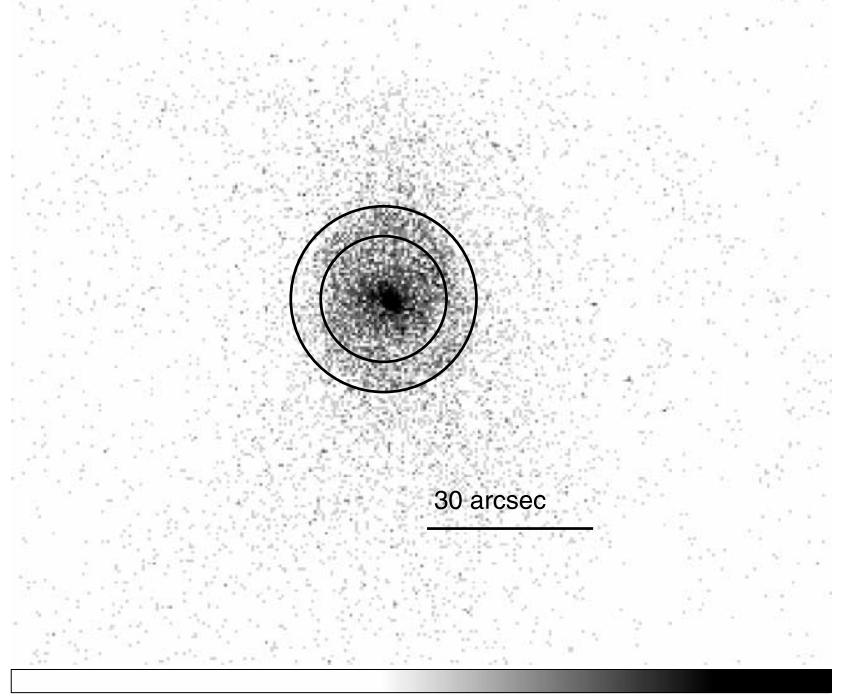

FIG. 2.-Background-subtracted Chandra image of the $0.5-2 \mathrm{keV}$ diffuse emission from the inner rings in the central region of NGC 4552 with spectral region $\mathrm{R}$ superposed; 1 pixel $=0$ "' $49 \times 0$ 0" 49 . [See the electronic edition of the Journal for a color version of this figure.]

CIAO version 3.1, FTOOLS, and XSPEC version 11.2. Filtering removed events with bad grades $(1,5$, and 7$)$ and those with significant flux in border pixels of the $5 \times 5$ event islands (VFAINT mode), as well as any events falling on hot pixels. The data were reprocessed and response files created using the most recent gain tables and instrumental corrections, including corrections for the time-dependent declining efficiency of the ACIS detector due to the buildup of contaminants on the optical filter (Plucinsky et al. 2003) and for the slow secular drift (tgain) of the average pulse-height amplitude values for photons of fixed energy. ${ }^{2}$ Periods of anomalously high background (flares) were identified and removed, along with times of anomalously low count rates at the beginning and end of the observation, resulting in a useful exposure time of $51,856 \mathrm{~s}$. Point sources were identified in the S3 chip in the $0.3-10 \mathrm{keV}$ energy band using a multiscale wavelet decomposition algorithm set with a $5 \sigma$ detection threshold. The resulting 132 source identifications were excluded from the spectral and surface brightness analyses of NGC 4552 here and in Paper I.

\section{NUCLEAR OUTFLOWS IN NGC 4552}

In Figure 1 we show the $0.7-1 \mathrm{keV}$ diffuse emission from the two ringlike features in the central region of NGC 4552. Point sources were removed from the image and the point source regions filled with the local average emission level using CIAO tool dmf ilth. In the right panel of Figure 1 we subtract the $0.7-1 \mathrm{keV}$ image smoothed with a 5".4 Gaussian kernel from the same image smoothed with a 1" Gaussian kernel to produce an unsharp masked image of the same region, in which these rings are clearly visible. The rings are approximately circular and of equal size with radii $\sim 0.85 \mathrm{kpc}\left(11^{\prime \prime}\right)$ and farthest projected distance from the nucleus of NGC 4552 of $\sim 1.3 \mathrm{kpc}\left(\sim 17^{\prime \prime}\right)$, creating in projection the "hourglass" appearance noted by Filho et al. (2004). Such cavities have been shown in other elliptical galaxies, such as M87 (Young et al. 2002; Forman et al. 2005), to chronicle past episodes of nuclear activity from a central AGN.

\footnotetext{
${ }^{2}$ See http://cxc.harvard.edu/contrib/alexey/tgain/tgain.html.
}

Spectral Analysis Regions for the Central 1.3 kpe of NGC 4552

\begin{tabular}{lccccc}
\hline \hline Region & Type & Shape & R.A. & Decl. & $\begin{array}{c}\text { Dimensions } \\
(\operatorname{arcsec})\end{array}$ \\
\hline R............. & $\begin{array}{c}\text { Source } \\
\text { R........... }\end{array}$ & $\begin{array}{c}\text { Background } \\
\text { Annular }\end{array}$ & 123539.9 & 123323.4 & 11,17 \\
NS ........... & $\begin{array}{c}\text { Source } \\
\text { NS ........... }\end{array}$ & Circular & 123539.9 & 123323.4 & 20,31 \\
\hline
\end{tabular}

Notes.-WCS coordinates for the centers of the regions are J2000.0. Dimensions specified are radii for circular regions, and (inner, outer) radii for annular regions. Region $\mathrm{R}$ is also shown Fig. 1.

\subsection{Gas Temperature in the Rings}

We fit the spectrum of the rings in an annular region $R$, shown in Figure 2 and listed in Table 1, with inner and outer radii of $11^{\prime \prime}$ and $17^{\prime \prime}$, respectively. The local background region for the rings (also listed in Table 1) was chosen concentric to and immediately outside the source to remove contamination from galaxy emission at larger radii as well as the Virgo ICM. We use an absorbed APEC thermal plasma model (Smith et al. 2001) corrected for absorption using Wisconsin photoelectric cross sections (Morrison \& McCammon 1983), with the absorbing column fixed at the Galactic value of $2.59 \times 10^{20} \mathrm{~cm}^{-2}$ (Dickey \& Lockman 1990), to fit the resulting 1840 net source counts over the $0.3-3 \mathrm{keV}$ energy range, where the source count rate was significantly above background. We allowed the hydrogen column to vary and found no suggestion for increased absorption above the Galactic value. We checked that there were no significant differences between the spectra for the northern and southern rings by fitting the northern and southern halves of region $\mathrm{R}$ separately. We found the temperature and abundance of the northern (southern) ring to be $0.64 \pm 0.03 \mathrm{keV}\left(0.65_{-0.04}^{+0.03} \mathrm{keV}\right)$ and $0.6_{-0.3}^{+0.4} Z_{\odot}\left(>0.5 Z_{\odot}\right)$, respectively, for fixed Galactic absorption. Since the spectral fits for the northern and southern rings agreed within errors, we combined these regions into the single annular region $\mathrm{R}$ to improve statistics.

The best-fit APEC model spectral parameters for gas in the rings from this combined annular region (region $\mathrm{R}$ ) are listed in the first row of Table 2 . We find the temperature and abundance of gas in the rings to be $k T=0.64 \pm 0.02 \mathrm{keV}$ and $A=0.7_{-0.2}^{+0.4} Z_{\odot}$ $\left(\chi^{2} /\right.$ dof $\left.=46 / 55\right)$, respectively. Thus, the temperature of gas in the rings (region $\mathrm{R}$ ) is a factor of 1.4 higher than that found for the surrounding $\left(0.43_{-0.02}^{+0.03} \mathrm{keV}\right)$ galaxy gas (Paper I). This is in contrast to the properties of a cold front, where the brighter

TABLE 2

Spectral Models of NGC 4552's Rings

\begin{tabular}{|c|c|c|c|c|}
\hline $\begin{array}{c}k T \\
(\mathrm{keV})\end{array}$ & $\begin{array}{c}A \\
\left(Z_{\odot}\right)\end{array}$ & $\begin{array}{c}K \\
\left(10^{-5} \mathrm{cgs}\right)\end{array}$ & $\begin{array}{c}\Lambda \\
\left(10^{-23} \operatorname{ergs~cm}^{3} \mathrm{~s}^{-1}\right)\end{array}$ & $\chi^{2 / d o f}$ \\
\hline $0.64 \pm 0.02$. & $0.7_{-0.2}^{+0.4}$ & 5.8 & 1.7 & $45.8 / 55$ \\
\hline $0.65_{-0.03}^{+0.02} \ldots \ldots$ & $0.4^{-0.2}$ & 9.1 & 1.1 & $55 / 56$ \\
\hline $0.64_{-0.02}^{+0.03} \ldots \ldots \ldots \ldots \ldots$ & 0.5 & 7.6 & 1.3 & $48.8 / 56$ \\
\hline $0.64_{-0.02}^{+0.03} \ldots \ldots \ldots \ldots \ldots \ldots$ & 0.6 & 6.6 & 1.5 & $46.4 / 56$ \\
\hline $0.64 \pm 0.02 \ldots \ldots \ldots \ldots \ldots$ & 0.8 & 5.1 & 1.9 & $46 / 56$ \\
\hline $0.64 \pm 0.02 \ldots \ldots \ldots \ldots$ & 1.0 & 4.2 & 2.4 & $47.4 / 56$ \\
\hline
\end{tabular}

NoTES.-Spectral model parameters for the rings from region $\mathrm{R}$ (see Table 1 and Fig. 1), using an absorbed single temperature APEC model fit over the $0.3-3 \mathrm{keV}$ energy range. The spectrum contains 1840 net source counts. The hydrogen absorption column is fixed at the Galactic value $\left(n_{\mathrm{H}}=2.59 \times 10^{20} \mathrm{~cm}^{-2}\right)$. The first row denotes the best-fit model with temperature and abundance free to vary, while following rows hold the abundance fixed. Errors are $90 \%$ confidence limits. 


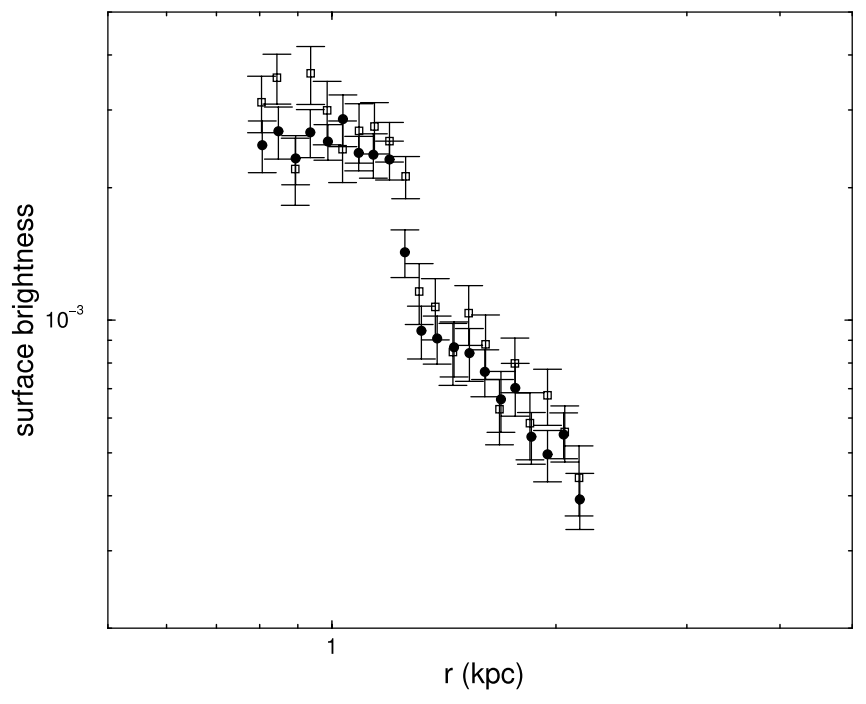

FIG. 3. - The $0.5-2 \mathrm{keV}$ surface brightness profiles across the northern (open squares) and southern ( filled circles) rings taken in angular sectors, whose vertices are positioned at the nucleus and angles extend from $64^{\circ}$ to $121^{\circ}$ for the northern rim and from $257^{\circ}$ to $349^{\circ}$ for the southern rim (measured counterclockwise from the west in Fig. 1).

region is cooler than its less bright surroundings (Viklinin et al. 2001; see also Paper I and references therein) or to the cool, bright rims of highly evolved remnant cavities from nuclear activity, such as those found in Perseus (Fabian et al. 2003) and M87 (Forman et al. 2005), but is similar to the properties observed in the X-ray edges produced by shocks driven into the ambient medium from recent AGN outbursts, such as in Hercules A (Nulsen et al. 2005a) and in Hydra A (Nulsen et al. 2005b).

We verified that the temperature rise in the rings cannot be due to unresolved X-ray binaries in our data by adding a $5 \mathrm{keV}$ bremsstrahlung component to our spectral model, as in Paper I, and refitting the spectrum over the $0.3-5 \mathrm{keV}$ energy range. The resulting best-fit temperature $\left(0.63_{-0.02}^{+0.03} \mathrm{keV}\right)$ for the thermal component in this two-component model agrees with the singlecomponent APEC model results to within $2 \%$. The abundance of the gas $\left(A=0.9_{-0.3}^{+0.5} Z_{\odot}\right)$ is also in agreement, within the $90 \%$ CL model uncertainties, with that found using a single APEC model. The $0.5-2 \mathrm{keV} \mathrm{X}$-ray luminosity of region $\mathrm{R}$ is dominated by emission from galaxy gas, with the bremsstrahlung (unresolved X-ray binary) component contributing $<5 \%$ to the $0.5-2 \mathrm{keV}$ luminosity in that region. This is in agreement with expectations for the residual contribution of unresolved X-ray binaries obtained from a direct integration of the average X-ray luminosity function for LMXBs (Gilfanov 2004) below our 0.5$8 \mathrm{keV}$ point source detection threshold $\left(4.8 \times 10^{-15} \mathrm{ergs} \mathrm{s}^{-1} \mathrm{~cm}^{-2}\right)$ for region $\mathrm{R}$. Thus, the effects of unresolved X-ray binaries on the measurement of the properties of the diffuse gas in the rings that are important for our analysis are small.

Although the uncertainties are large, the metallicity in the rings is consistent with that found in Paper I for the outer regions of the galaxy. Thus, we find no evidence for a strong metallicity gradient across the rings. For completeness we list in Table 2 the metallicity dependence of the $0.5-2 \mathrm{keV} \mathrm{X}$-ray emissivity for gas in the rings over the abundance range $\left(0.4-1.0 Z_{\odot}\right)$ of interest for NGC 4552.

\subsection{Gas Density in the Rings}

As shown in Figure 3, the $0.5-2 \mathrm{keV}$ surface brightness profiles across the northern and southern rims, taken in an angular sector centered on the nucleus extending from $64^{\circ}$ to $121^{\circ}$ for the northern rim and $257^{\circ}$ to $349^{\circ}$ for the southern rim (measured counterclockwise from west in Fig. 1), agree within errors. Thus, we use the surface brightness profile across the northern rim as representative of the ring system. We use the multivariate minimization technique (Markevitch et al. 2000; Vikhlinin et al. 2001) to determine the electron density inside the rim of the northern ring from the surface brightness profile shown in Figure 4.

We assume a spherically symmetric power-law distribution for the electron density for $r \leq r_{1}$ of the form

$$
n_{e}=n_{\text {rim }}\left(\frac{r}{r_{1}}\right)^{-\alpha_{1}}
$$

where $r_{1}$ is the outer radius (rim) of the ring, and $n_{\text {rim }}$ and $\alpha_{1}$ are the normalization and power-law index, respectively, for the electron density of NGC 4552 inside the rim of the northern ring.
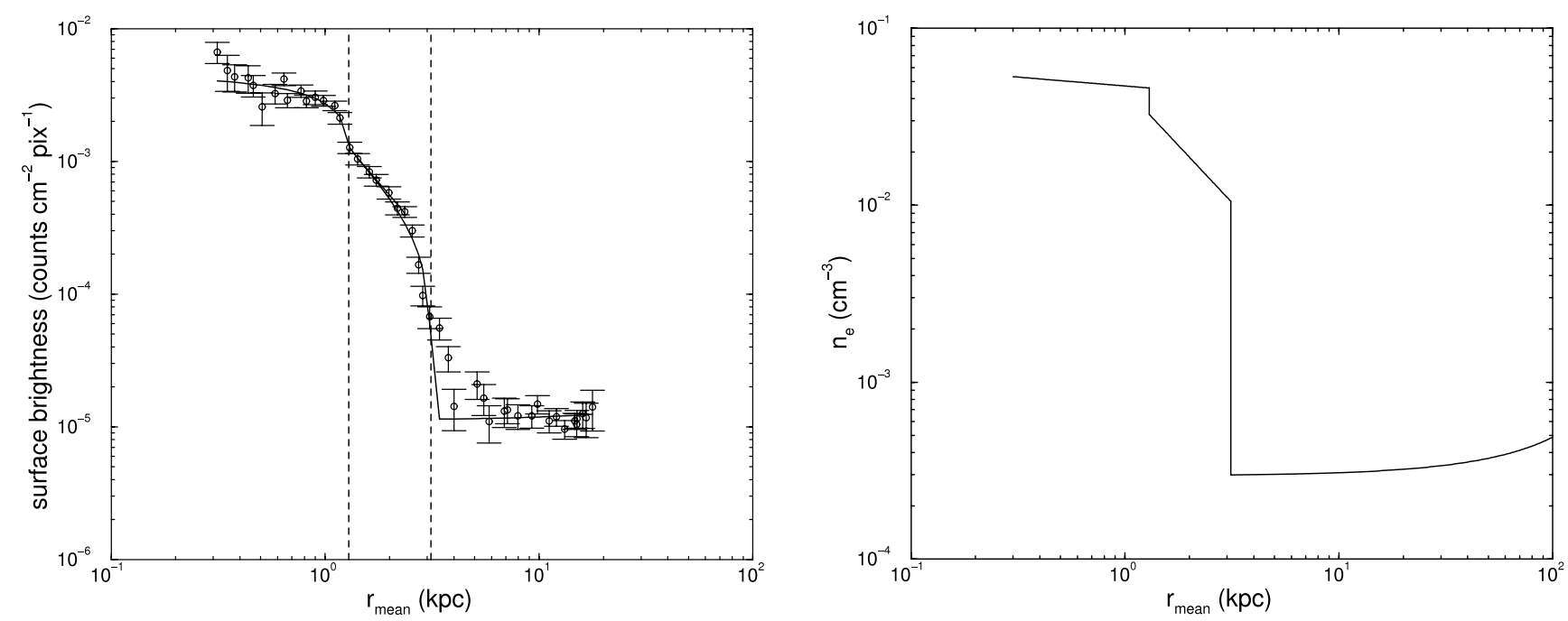

FIG. 4. - Left: $0.5-2 \mathrm{keV}$ surface brightness profile as a function of distance from the center of NGC 4552 toward the north across the galaxy's leading edge from Paper I. The vertical dashed lines denote $r_{1}=1.3 \mathrm{kpc}$ and $r_{2}=3.1 \mathrm{kpc}$, the position of the outer radius (rim) of the northern ring and the outer leading edge of the cold front, respectively. The solid line denotes the model results for the surface brightness for power-law density models $n_{e} \propto r^{-\alpha}$ within the galaxy with $\alpha=0.1_{-0.1}^{+0.25}$ for $r<1.3 \mathrm{kpc}$ (inside the rim of the ring) and $\alpha=1.3_{-0.2}^{+0.2}$ for $1.3 \leq r<3.1 \mathrm{kpc}$ (between the rim of the ring and the leading edge of the cold front). Right: Electron density as a function of radius from the center of NGC 4552 for the above model. 
The density model for NGC 4552's gas outside the ring $r_{1} \leq$ $r \leq r_{2}$, determined by our analysis of the northern leading edge in Paper I, is given by

$$
n_{e}=n_{e 2}\left(\frac{r}{r_{2}}\right)^{-\alpha_{2}}
$$

where $r_{2}=3.1 \mathrm{kpc}, \alpha_{2}=1.3 \pm 0.2$, and $n_{e 2}=0.01 \mathrm{~cm}^{-3}$. Since NGC 4552 is much smaller than the projected 71.'6 separation between M87 and NGC 4552, the contribution of the cluster ICM to the surface brightness for radii $r \leq 3.1 \mathrm{kpc}$ (inside the galaxy) is nearly constant, varying by $\$ 2 \%$. We subtract this ICM contribution from the surface brightness profile within NGC 4552 $(r<3.1 \mathrm{kpc})$ and fit the remaining galaxy emission, taking the position $r_{\text {rim }}$ of the rim of the ring, the galaxy electron density power-law indices $\alpha_{1}, \alpha_{2}$, and the discontinuity across the rim as free parameters. The discontinuity across the rim is given by

$$
J=\left(\frac{\Lambda_{\text {rim }} n_{\text {rim }}^{2}}{\Lambda_{\text {out }} n_{\text {out }}^{2}}\right)^{1 / 2},
$$

where $n_{\text {rim }}$ and $\Lambda_{\text {rim }}$ denote the electron density and emissivity inside the rim of the northern ring, and $n_{\text {out }}$ and $\Lambda_{\text {out }}$ denote the corresponding properties of galaxy gas outside the rim.

We find a best-fit position for the discontinuity across the rim shown in Figure 4 of $r_{1}=1.30 \mathrm{kpc}$, coincident with the rim (outer radius) of the northern ring in Figure 1, and slope $\alpha_{1}=0.1 \pm 0.3$ for the electron density at $r \leq r_{1}$. The slope of the electron density distribution for radii $r>r_{1}$ (outside the rings) was $\alpha_{2}=$ $1.3 \pm 0.2$, consistent with our previous fit in Paper I for the galaxy gas at large radii. Assuming no strong abundance gradients in NGC 4552, and correcting for the $20 \%$ difference in the galaxy gas cooling functions caused by the difference in gas temperature between gas at large radii $(0.43 \mathrm{keV}$, Paper I) and gas in the rings $(0.64 \mathrm{keV}$ for region $\mathrm{R})$, we infer the density ratio across the rim of the northern ring to be $n_{\mathrm{rim}} / n_{\text {out }}=1.4_{-0.2}^{+0.3}$. We extrapolate equation (2) to $r=1.3 \mathrm{kpc}$, the location of the rim of the northern ring, to evaluate $n_{\text {out }}$ at the discontinuity. Then using the density discontinuity at the rim, we find the electron density just inside the rim to be $n_{\text {rim }}=0.05 \mathrm{~cm}^{-3}$. In the right panel of Figure 4, we plot the resulting normalized two power-law density model for gas in NGC 4552 and the $\beta$-model for the surrounding Virgo ICM (Schindler et al. 1999) as a function of distance from the center of NGC 4552.

\subsection{Shocks from Recent Nuclear Activity}

Since the temperature $\left(k T_{\text {rim }}=0.64 \mathrm{keV}\right)$ and density $\left(n_{\text {rim }}=\right.$ $0.05 \mathrm{~cm}^{-3}$ ) of gas in the inner rings are both greater than that for galaxy gas outside the rings $\left(k T_{\text {out }} \sim 0.43 \mathrm{keV}, n_{\text {out }} \sim 0.03 \mathrm{~cm}^{-3}\right.$ ) at $r_{1}=1.3 \mathrm{kpc}$, the properties of the rings are qualitatively consistent with those resulting from a shock. For a monatomic ideal gas with adiabatic index $\gamma$, we can use the density discontinuity $\rho_{\text {rim }} / \rho_{\text {out }}=n_{\text {rim }} / n_{\text {out }}$ in the Rankine-Hugoniot shock conditions (Landau \& Lifschitz 1959) across the shock to estimate the shock speed (Mach number $M_{1}$ )

$$
\frac{n_{\text {rim }}}{n_{\text {out }}}=\frac{(\gamma+1) M_{1}^{2}}{(\gamma-1) M_{1}^{2}+2}
$$

and predict discontinuities in temperature

$$
\frac{T_{\text {rim }}}{T_{\text {out }}}=\frac{\left[2 \gamma M_{1}^{2}-(\gamma-1)\right]\left[(\gamma-1) M_{1}^{2}+2\right]}{(\gamma+1)^{2} M_{1}^{2}}
$$

and pressure

$$
\frac{p_{\text {rim }}}{p_{\text {out }}}=\frac{2 \gamma M_{1}^{2}}{\gamma+1}-\frac{\gamma-1}{\gamma+1} .
$$

We can obtain an estimate for the density jump $n_{\text {rim }} / n_{\text {out }}$ from the surface brightness discontinuity at the position of the rings. However, since the shock front is narrow, the averaged, measured surface brightness discontinuity will underestimate the actual density discontinuity at the narrow shock front and thus also the inferred shock strength and temperature rise. From our fit to the inner surface brightness edge in $\S 3.2$, we infer a lower limit on the density discontinuity of $1.4_{-0.2}^{+0.3}$. From equation (4) this implies a shock Mach number $M_{1} \gtrsim 1.3$. For this Mach number, the temperature and pressure increases predicted by equations (5) and (6) are $T_{\text {rim }} / T_{\text {out }} \gtrsim 1.3$ and $p_{\text {rim }} / p_{\text {out }} \gtrsim 1.8$, in excellent agreement with our measured ratios of $1.48_{-0.13}^{+0.15}$ and $2.1_{-0.5}^{+0.7}$, respectively. Thus, the bright rings in Figure 1 are shocks at the interface between the surrounding galaxy gas and two expanding cavities. Numerical simulations have predicted that such features would be formed by high-momentum, subrelativistic bipolar nuclear outflows (see, e.g., Reynolds et al. 2001; Omma et al. 2004 and references therein).

An estimate for the age of these cavities is given by $t_{\text {age }} \sim R / c_{s}$ where $R$ is the mean radius of the cavity and $c_{s}$ is the sound speed in the ambient medium (Birzan et al. 2004). For $R \sim 0.85 \mathrm{kpc}$ and $c_{s} \sim 340 \mathrm{~km} \mathrm{~s}^{-1}$, the speed of sound in $0.43 \mathrm{keV}$ galaxy gas, we find the cavities to be $\sim 2.4 \mathrm{Myr}$ old. A lower limit on the total mechanical energy $E_{\text {mech }}$ carried in the outflow is given by the work $2 p_{g} V$ needed to evacuate the two observed cavities, where $p_{g}$ is the mean pressure of the unshocked galaxy gas at each cavity's center and $V$ is the volume of the cavity. Assuming spherical symmetry for each expanding cavity and extrapolating the density model in equation (2) for unshocked galaxy gas outside the rings to the center of each cavity, we find the sum of the mechanical energy for the two cavities to be $E_{\text {mech }}>1.2 \times 10^{55}$ ergs. This gives a lower limit on the average mechanical power for the outburst of $L_{\text {mech }}>1.5 \times 10^{41} \mathrm{ergs} \mathrm{s}^{-1}$.

We use a simple, spherically symmetric, point explosion shock model to obtain a second estimate for the properties of the outflow. The details of this model and its uncertainties are discussed in Nulsen et al. (2005a, 2005b). We briefly summarize that discussion here. The model shock is produced by a sudden deposit of energy in the central cell of the simulation. The model then uses gas hydrodynamical equations, including gravity, to numerically propagate the shock radially outward through the galaxy gas, assuming the unshocked galaxy atmosphere is isothermal, spherically symmetric with a power-law density distribution, and in hydrostatic equilibrium. Assuming the gas in NGC 4552 is in hydrostatic equilibrium, we use our fits to the outer region (OA in Paper I) of NGC 4552 as representative of the temperature $(k T=0.43 \mathrm{keV})$ and density distribution $\left(\propto r^{-1.3}\right)$ of the initially unshocked galaxy gas. We then compute surface brightness profiles from the model, using the Chandra response in the $0.5-2 \mathrm{keV}$ energy band, determined using XSPEC and an absorbed APEC model for temperatures determined from the shock model, assuming a preshock temperature of $k T=0.43 \mathrm{keV}$ and detector responses appropriate to this observation. Since the shock weakens as it hydrodynamically evolves, and the initial conditions are self-similar, we scale the model flow radially to the position of the observed shock. We then match the observed surface brightness profile to that of the model to determine the shock parameters. Although this point explosion model will not reproduce the detailed (bipolar) geometry of the outflows, the model does provide a good description for the shape of the 

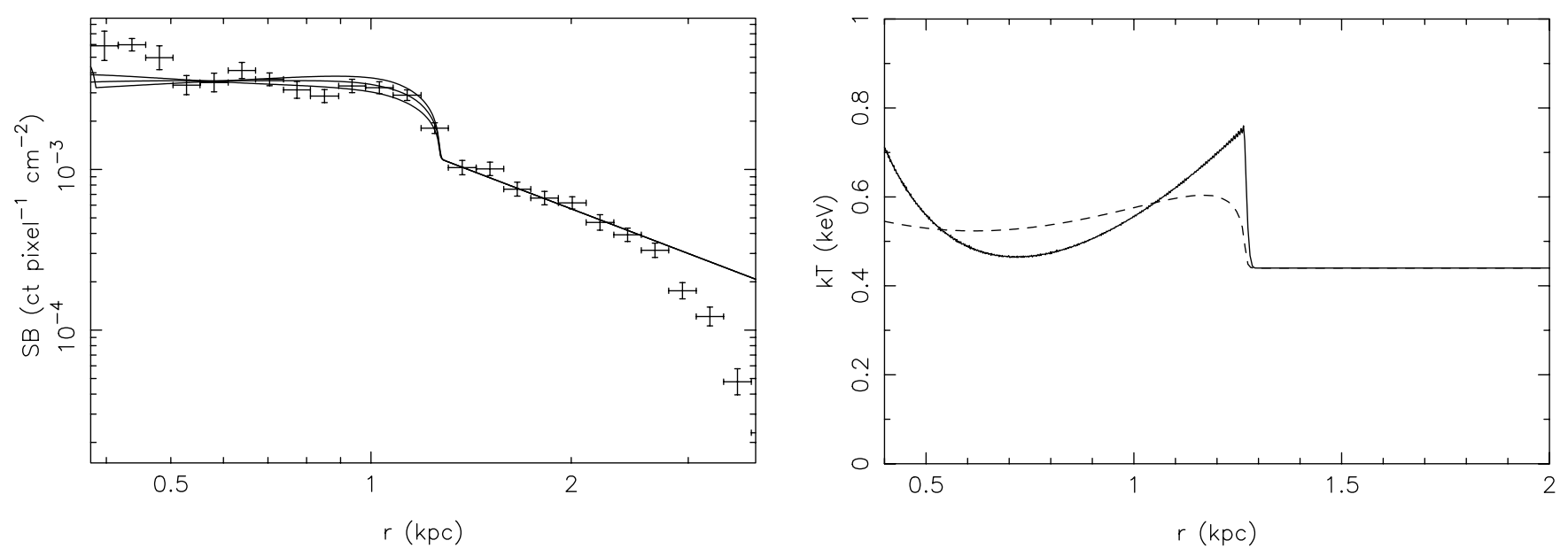

FIG. 5. - Left: Spherical shock model fits to the surface brightness profile across the inner edge for a preshocked isothermal $(0.43 \mathrm{keV}) r^{-1.3}$ density distribution inside the galaxy. Top, middle, and bottom lines represent shock Mach numbers 1.9, 1.7, and 1.6, respectively. Right: Temperature profile (solid line) for the Mach 1.7 model shock shown in the left panel. Dashed line denotes the emission-measure-weighted temperature profile for lines of sight through the galaxy.

surface brightness profile across the rim and thus a reasonable measure of the Mach number, and thus temperature jump, of the shock (Nulsen et al. 2005b).

In the left panel of Figure 5 we see that the surface brightness profile across the rim of the northern ring in NGC 4552 is well represented by a shock model with Mach number $M_{1}=1.7_{-0.1}^{+0.2}$ and shock radius of $1.29 \mathrm{kpc}$. The predicted, deprojected temperature profile across the shock front for the $M_{1}=1.7$ model is shown as the solid line in the right panel of Figure 5 . The radial extent of region $\mathrm{R}(0.9 \lesssim r \lesssim 1.29 \mathrm{kpc})$, used to measure the temperature inside the rings, is much larger than the radial width of the shock. Our measured temperature $(0.64 \pm 0.02 \mathrm{keV}$ from Table 2) represents an average over this region and is in good agreement with the model results. Projection effects also tend to reduce the observed temperature, since any given line of sight through the shock also passes through surrounding unshocked galaxy gas of lower temperature. The dashed line in the right panel shows the predicted emission-measure-weighted temperature profile $T_{\text {proj }}$ for our Mach 1.7 model, taking into account the contribution from cooler preshocked galaxy gas along the line of sight. The emission-measure-weighted temperature profile rises sharply at the shock boundary, peaking at $\sim 0.6 \mathrm{keV}$, and then slowly decreases to $\sim 0.55 \mathrm{keV}$ at $r=0.9 \mathrm{kpc}$, the inner radius of region $\mathrm{R}$. This predicted profile is shifted up or down by $\sim 0.03 \mathrm{keV}$ due to uncertainties in the temperature of the preshocked galaxy gas. Since the model $T_{\text {proj }}$ includes the contribution of unshocked galaxy gas at radii larger than that of the rings that we subtracted as a local background in our previous fits to region $\mathrm{R}$, we refit the spectrum for the rings in region $\mathrm{R}$ for comparison with the model, using a $6.7 \times 2 .{ }^{\prime} 5$ rectangular region (region VN in Paper I) centered at $\alpha=12^{\mathrm{h}} 35^{\mathrm{m}} 37^{\mathrm{s}} .7$, $\delta=12^{\circ} 36^{\prime} 16^{\prime \prime} .5$, with an orientation angle of $319^{\circ}$ comprising approximately $25 \%$ of the area of the $\mathrm{S} 3$ chip as the local background, to subtract only the Virgo ICM and particle backgrounds from the data. We find a mean temperature $k T_{\text {proj }}=0.61 \pm 0.02 \mathrm{keV}$ and abundance $A=0.6 \pm 0.15\left(\chi^{2} / \mathrm{dof}=51.8 / 55\right)$ for fixed Galactic absorption. This is in excellent agreement with the shock model prediction (Fig. 5, just behind the shock). We verified that this result was not biased by our choice of local background by refitting the spectrum using blank sky backgrounds appropriate for the date of observation and instrument configuration. ${ }^{3}$

\footnotetext{
${ }^{3}$ See http://cxc.harvard.edu/contrib/maxim/acisbg and Paper I.
}

When we used a second APEC component to model the contribution of the Virgo Cluster gas, with temperature $2.2 \mathrm{keV}$ and abundance $0.1 Z_{\odot}$ fixed at the "best-fit" Virgo Cluster values from Paper I but with the normalization free to vary, we found no significant differences.

The bipolar geometry of the outburst cavities, seen most clearly in the right panel of Figure 1, makes it difficult to probe the temperature profile inside the rims. To investigate this region, we extract a combined spectrum from elliptical regions in the northern (southern) ring centered at $\alpha=12^{\mathrm{h}} 35^{\mathrm{m}} 39^{\mathrm{s}} \mathrm{s} 7, \delta=$ $+12^{\circ} 33^{\prime} 31^{\prime \prime} 7\left(\alpha=12^{\mathrm{h}} 35^{\mathrm{m}} 39^{\mathrm{s}} 53, \delta=+12^{\circ} 33^{\prime} 15^{\prime \prime}\right.$. 97$)$ with semimajor, minor axes, and position angle ( $a, b$, P.A.) of $a=0.47 \mathrm{kpc}$, $b=0.27 \mathrm{kpc}$, P.A. $=9.5(a=0.52 \mathrm{kpc}, b=0.20 \mathrm{kpc}$, P.A. $=$ 21.3 ), respectively. We use these elliptical regions rather than a simple annular region inside the rings in order to avoid the region just outside the nucleus where, in projection, the bipolar ring rims appear to intertwine. We model the spectrum in XSPEC with an absorbed two-component APEC model, fixing the temperature and abundance of one APEC component at the temperature and abundance of the Virgo Cluster gas, as above, and hydrogen absorption at the Galactic value. We find a mean temperature and abundance for galaxy gas averaged along the lines of sight passing through the interior of the cavities of $k T_{\text {proj }}=0.65_{-0.04}^{+0.03} \mathrm{keV}$ and $A=0.6_{-0.2}^{+0.4} Z_{\odot}\left(\chi^{2} / \mathrm{dof}=43 / 34\right)$, respectively. This agrees within $90 \%$ confidence with the mean temperature and abundance $\left(k T_{\text {proj,rim }}=0.61 \pm 0.02 \mathrm{keV}, A=0.6 \pm 0.15 Z_{\odot}\right)$ measured in the annular ring region $\mathrm{R}$.

Thus, we do not observe the $\$ 10 \%$ decline in temperature behind the shock that might have been expected from the model temperature profile in the right panel of Figure 5. This is not surprising for several reasons. First, both the ring and cavity spectral regions average over a broad range in radius. Region $\mathrm{R}$, with inner and outer radii of 0.9 and $1.3 \mathrm{kpc}$, averages over $30 \%$ of the radial distance from the shock rim to the nucleus, thus already averaging over the region where the temperature decline due to adiabatic expansion of gas behind the shock front should be greatest. Similarly, the elliptical regions for the cavities also sample a broad range in radius that is further complicated by projection effects due to the bipolar geometry of the outflows. Second, the model evolution of the gas temperature behind the shock front is sensitive to the density and temperature profile of the unshocked atmosphere. The unshocked gas density in galaxies typically follows a $\beta$-model profile, flattening in the core. 
Thus, it is strictly a power law only locally near the rim. Also, galaxy gas near the nucleus of NGC 4552 may not be isothermal. Finally, the outflows are likely still driven by the ram pressure of jet plasma from the initial AGN outburst that has effectively evacuated X-ray gas from a "cocoon" inside the shocked rims, rather than by a point explosion model. This would also affect the observed thermal properties of gas averaged over lines of sight passing through the cocoon. In particular, if the cavities are nearly devoid of X-ray gas, the emission will be dominated by the front and back edges of the cavities. Additional discussion of these model uncertainties can be found in Nulsen et al. (2005b).

Nonetheless, the model does fit the surface brightness profile in the vicinity of the shock well (see Fig. 5), such that the shock Mach number is largely insensitive to the details of the model. Consequently, the point explosion model estimates for the total mechanical energy and mean mechanical power carried in such outbursts are unlikely to err from their true values by more than a factor of $\sim 2$ (Nulsen et al. 2005b). Adopting a factor of 2 as a conservative estimate for these model uncertainties, we now use the shock model fit to our data to determine the total mechanical energy and mean mechanical power required for the nuclear outburst observed in NGC 4552. The elapsed time since the nuclear outburst (shock age) in the Mach 1.7 spherical shock model is $1.3 \mathrm{Myr}$. Since the simplified spherical model geometry overestimates the volume swept out by the observed shocks, the model predictions for the total mechanical energy $E_{\text {mech }}$ carried in the shocks and thus also the mean mechanical power $L_{\text {shock }}$ of the outburst are upper bounds on the properties of the observed outburst in NGC 4552. We correct for this by including a geometrical factor $f_{V} \leq 1$, where $f_{V}$ is approximately the ratio of the volume filled by the observed cavities to that of the spherical model. For a Mach 1.7 shock, we find $E_{\text {mech }}=2.4 \times 10^{55} f_{V}$ ergs and $L_{\text {shock }}=$ $5.8 \times 10^{41} f_{V}$ ergs s$^{-1}$. Assuming the observed cavities are well approximated by two spheres of radius $R=0.85 \mathrm{kpc}$, we find that the fraction of the model volume filled by these cavities is $f_{V} \sim 0.6$, such that the total mechanical energy and mean mechanical luminosity of the outburst are $E_{\text {mech }} \sim 1.4 \times 10^{55}$ ergs and $L_{\text {shock }} \sim 3.3 \times 10^{41} \mathrm{ergs} \mathrm{s}^{-1}$, respectively, consistent with our previous estimate.

We obtain an estimate of the total X-ray cooling rate for the galaxy ISM by fitting a mean spectrum for NGC 4552 extracted from a $50^{\prime \prime}(3.8 \mathrm{kpc})$ circular region centered on the galaxy's nucleus, using a two-component absorbed APEC model with absorption fixed at the Galactic value and one set of parameters fixed at the best-fit Virgo Cluster parameters. We find a mean temperature $(0.57 \pm 0.01 \mathrm{keV})$, abundance $\left(0.5 \pm 0.1 Z_{\odot}\right)$, and $0.2-2 \mathrm{keV}$ flux of $F(0.2-2)=7 \times 10^{-13} \mathrm{ergs} \mathrm{s}^{-1} \mathrm{~cm}^{-2}$ for NGC 4552's ISM component, in excellent agreement with results $\left[k T=0.55_{-0.08}^{+0.11} \mathrm{keV}, F(0.2-2)=6.8_{-1.0}^{+1.1} \times 10^{-13} \mathrm{ergs} \mathrm{s}^{-1} \mathrm{~cm}^{-2}\right]$ by Matsushita (2001) using ROSAT data. Using the 0.1-14 keV luminosity as an estimate for the bolometric luminosity $L_{\mathrm{bol}}$ of the hot gas, we find $L_{\mathrm{bol}}=2.5 \times 10^{40} \mathrm{ergs} \mathrm{s}^{-1}$. If we use a more realistic two-component temperature model for the ISM, consistent with our spectral results for the individual regions, this estimate of the total flux decreases by $\lesssim 2 \%$. Therefore, the energy carried in the observed nuclear outflows $\left(E \sim 1.4 \times 10^{55} \mathrm{ergs}\right)$ is sufficient to balance cooling of the galaxy ISM for $\approx 18 \mathrm{Myr}$.

Energetic outbursts could either be powered by supernovae from a compact circumnuclear region of star formation, i.e., in the central $\sim 100 \mathrm{pc}$ (1."3 radius) of the galaxy, or by an AGN at the galaxy's center. Assuming $10^{51}$ ergs kinetic energy per supernova and $\sim 1$ supernova per $100 M_{\odot}$ of star formation (McNamara et al. 2004; Silk et al. 1986), we would need $\gtrsim 14,000$ supernovae to provide the outburst energy of $1.4 \times 10^{55} \operatorname{ergs}$ (for $f_{V}=0.6$ ). This would require a mean star formation rate, over the $1.3 \mathrm{Myr}$ age of the outburst, of $\gtrsim 1 M_{\odot} \mathrm{yr}^{-1}$ within $100 \mathrm{pc}$ of the nucleus. If we make the extreme assumption that all star formation in NGC 4552 occurs in this circumnuclear region, we can use the total UV emission of the galaxy at $2500 \AA$ as a direct tracer of light from unabsorbed star formation, and the TIR emission (IR emission integrated over the $8-1000 \mu \mathrm{m}$ band) as a tracer of star formation in obscured dusty regions, to place a firm upper bound on the energy from star formation, that is available to power the observed outbursts. Using NGC 4552's UV luminosity, calculated from photometric data at $2500 \AA$ (NED) in equation (1) from Kennicutt (1998), we find an unobscured star formation rate of $0.09 M_{\odot} \mathrm{yr}^{-1}\left(0.14 M_{\odot} \mathrm{yr}^{-1}\right)$ for a continuous (starburst) star formation model. This is an order of magnitude below that needed to power the observed outburst. Note that the unobscured star formation rate in the inner $20 \mathrm{pc}$ nuclear region calculated using the UV flux measured by Maoz et al. (2005) is very small, $<0.0002-0.0003 M_{\odot} \mathrm{yr}^{-1}$. This is consistent with the high brightness temperature and flat spectral shape of NGC 4552's radio emission that also argue against a dominant compact nuclear starburst or collection of supernova remnants in the galaxy's core (Nagar et al. 2002).

The TIR luminosity provides a tracer of star formation in dusty regions, where the UV emission from young stars has been reprocessed to longer wavelengths by the surrounding dust. Since dust in elliptical galaxies tends to be centrally concentrated, and dust has been identified in the inner $60 \mathrm{pc}$ of NGC 4552 with HST observations (van Dokkum \& Franx 1995), this is likely a reasonable estimate for obscured star formation in the inner $100 \mathrm{pc}$ of interest. We use the 60 and $100 \mu \mathrm{m}$ FIR flux densities for NGC 4552 from NED to calculate the FIR luminosity (Wise et al. 1993). We convert the FIR luminosity into the TIR luminosity using a correction factor [ $\leqslant 2$ for $F(60 \mu \mathrm{m}) / F(100 \mu \mathrm{m})=0.3$; Helou et al. 1988]. Then, using equation (4) in Kennicutt (1998), we find the star formation rate for obscured regions in NGC 4552 to be $\lesssim 0.03 M_{\odot} \mathrm{yr}^{-1}$. Summing the UV and TIR star formation rates, we find a firm upper limit for circumnuclear star formation of $\lesssim 0.12-0.17 M_{\odot} \mathrm{yr}^{-1}$, more than a factor of 5 below that needed to power the outburst. These low upper limits on current star formation in NGC 4552 are consistent with the 9.6 Gyr average age of the galaxy's stellar population determined from optical line ratios by Terlevich \& Forbes (2002). Thus, the outburst is most likely powered by NGC 4552's central AGN.

VLBA measurements (Nagar et al. 2002) show that NGC 4552 has a core radio source with two-sided east-west extended emission on $\sim 5$ mas scales. The radio spectrum is flat, with spectral index $\sim 0$ (Filho et al. 2004), and radio flux densities of 102.1 and $99.5 \mathrm{mJy}$ at 8.4 and $5 \mathrm{GHz}$, respectively. Integrating this radio flux over the $10-5000 \mathrm{MHz}$ frequency band, we find a total central radio power of $L_{\text {radio }}=1.55 \times 10^{38} \mathrm{ergs} \mathrm{s}^{-1}$. Filho et al. (2004) argue that the properties of NGC 4552's radio spectrum are most consistent with emission from accretion onto a $\sim 4 \times 10^{8} \mathrm{M}_{\odot}$ black hole plus a self-absorbed compact jet or outflow. Our observation of shocks produced by outflows from a previous episode of nuclear activity supports this model.

Furthermore, the mechanical luminosity, $L_{\text {shock }} \sim 3.3 \times$ $10^{41} \mathrm{ergs} \mathrm{s}^{-1}$ (with factor of $\sim 2$ uncertainty), found from our model fit to the observed shock in NGC 4552, is consistent with mechanical luminosities inferred for cavities produced by AGN outbursts in other systems. Using 18 systems, ranging in size from galaxy clusters to the elliptical galaxy M84 and containing well-defined X-ray surface brightness depressions (cavities), 
TABLE 3

Spectral Models of the Nuclear Region of NGC 4552

\begin{tabular}{ccccc}
\hline \hline $\begin{array}{c}n_{\mathrm{H}} \\
\left(10^{20} \mathrm{~cm}^{-2}\right)\end{array}$ & $\begin{array}{c}k T \\
(\mathrm{keV})\end{array}$ & $\begin{array}{c}A \\
\left(Z_{\odot}\right)\end{array}$ & $\alpha$ & $\chi^{2} / \mathrm{dof}$ \\
\hline $11 \pm 4 \ldots \ldots \ldots \ldots \ldots .$. & $\ldots .0 .13$ & $\ldots$ & $2.2 \pm 0.2$ & $51.7 / 45$ \\
$2.59^{\mathrm{a}} \ldots \ldots \ldots \ldots \ldots \ldots .$. & $1.04_{-0.17}^{+0.13}$ & $0.5_{-0.4}^{+0.9}$ & $1.7_{-0.3}^{+0.2}$ & $35.6 / 43$ \\
$2.59^{\mathrm{a}} \ldots \ldots \ldots \ldots \ldots \ldots$. & $1.04_{-0.11}^{+0.21}$ & $0.7^{\mathrm{a}}$ & $1.7 \pm 0.1$ & $35.7 / 44$ \\
$<6.7 \ldots \ldots \ldots \ldots \ldots \ldots$. & $1.04_{-0.18}^{+0.13}$ & $0.7^{\mathrm{a}}$ & $1.7 \pm 0.2$ & $35.6 / 43$ \\
\hline
\end{tabular}

Notes.-The spectrum contains 1046 net source counts in region NS, specfied in Table 1, over the $0.3-5 \mathrm{keV}$ band used for these fits. Errors are $90 \%$ confidence limits.

${ }^{\mathrm{a}}$ Fixed parameter.

Birzan et al. (2004) found a trend between the mechanical power $L_{\text {mech }}$ needed to create the X-ray cavities and the current central radio power $L_{\text {radio }}$ of the source that they parameterized as a power law

$$
L_{\text {mech }}=10^{25 \pm 3}\left(L_{\text {radio }}\right)^{0.44 \pm 0.06},
$$

albeit with large scatter. The error on the mechanical luminosity in equation (7) reflects the several orders of magnitude uncertainty due to scatter in their data. From equation (7), we expect the central radio source in NGC 4552 to produce outburst cavities with mechanical power $\log L_{\text {mech }}=41.8 \pm 3 \mathrm{ergs} \mathrm{s}^{-1}$, where again the errors reflect the uncertainty due to scatter in the data around the trend line. This is in good agreement with the mechanical power $\log L_{\text {shock }}=41.5 \pm 0.3 \mathrm{ergs} \mathrm{s}^{-1}$ found from our model fit to the observed shocks in NGC 4552, where the errors in our observed value reflect the factor of 2 uncertainty in the shock model results.

\section{THE NUCLEAR REGION}

Finally, we look briefly at the X-ray properties of the nuclear region by modeling the spectrum from a 1 ."3 circular region (NS) centered at ( $\alpha=12^{\mathrm{h}} 35^{\mathrm{m}} 39^{\mathrm{s}} .8, \delta=+12^{\circ} 33^{\prime} 22^{\prime \prime} .9$ ) containing the nuclear source (see Table 1). After local background subtraction using a concentric annular region with inner and outer radii of 1".6 and 3.2 , respectively, the spectrum contains 1046 net source counts in the $0.3-5 \mathrm{keV}$ energy band. An acceptable fit to the data $\left(\chi^{2} /\right.$ dof $\left.=52 / 45\right)$ was found using an absorbed power-law model with moderate hydrogen absorption $\left(n_{\mathrm{H}}=1.1 \pm 0.4 \times\right.$ $\left.10^{21} \mathrm{~cm}^{-2}\right)$ and steep $(\Gamma=2.2 \pm 0.2)$ photon index. The fits were improved and the photon index softened to $\Gamma=1.7 \pm 0.2$ (see Table 3) by adding a second, thermal APEC component to the model and fixing the hydrogen absorption for both thermal and power-law components at the Galactic value $\left(n_{\mathrm{H}}=2.59 \times\right.$ $10^{20} \mathrm{~cm}^{-2}$ ). For these two-component models, we first allow the temperature, metal abundance, and photon index to vary, but we find the abundance is poorly constrained. We then fix the metal abundance at $A=0.7 Z_{\odot}$ from the best-fit spectral model for the ring region R of NGC 4552 (see the first line in Table 2) and find that the fit is unchanged, although the errors on the photon index are modestly reduced. We checked the stability of this twocomponent fit by allowing the absorbing column to vary and found $n_{\mathrm{H}}$ consistent with the Galactic value.

In the two-component model, we find a photon index $\Gamma \sim$ $1.7 \pm 0.1$ and temperature $k T=1.04_{-0.17}^{+0.21} \mathrm{keV}$. The power-law photon index is consistent with those expected for low-luminosity AGNs (Terashima \& Wilson 2003; Filho et al. 2004). The tem- perature for the thermal component is higher than that found for gas in any other region of the galaxy, suggesting that we may be directly observing the reheating of the galaxy ISM by the nuclear outburst. We find intrinsic total $0.5-2$ and 2-10 keV X-ray fluxes in this model to be $5.1 \times 10^{-14}$ and $6.7 \times 10^{-14} \mathrm{ergs} \mathrm{s}^{-1} \mathrm{~cm}^{-2}$, corresponding to $0.5-2.0$ and $2-10 \mathrm{keV}$ luminosities of $1.6 \times 10^{39}$ and $2.1 \times 10^{39} \mathrm{ergs} \mathrm{s}^{-1}$, respectively. The power-law component dominates the emission in both energy bands, contributing $75 \%$ and $97 \%$ of the emission in the $0.5-2$ and $2-10 \mathrm{keV}$ bandpasses, respectively. These results are in good agreement with the spectral results of Filho et al. (2004) for a circular region 2 ..5 in diameter surrounding the nucleus with data extracted from the same Chandra observation (ObsID 2072) used in this work.

Since the nuclear source is known to be variable at the $\sim 20 \%$ level for the 2 and $3.6 \mathrm{~cm}$ radio flux on timescales $\sim 1 \mathrm{yr}$ (Nagar et al. 2002) and by a similar amount in the UV at 2500 and $3300 \AA$ on timescales of $\sim 2$ months (Maoz et al. 2005), it is interesting to look for X-ray variability. We examined the light curve for a $2^{\prime \prime}$ radius circular region centered on the nucleus $\left(\alpha=12^{\mathrm{h}} 35^{\mathrm{m}} 39^{\mathrm{s}} .8\right.$, $\delta=+12^{\circ} 33^{\prime} 22^{\prime \prime}$. 9 ) over the $0.3-10 \mathrm{keV}$ energy range using $3 \mathrm{ks}$ binning and found that, on these timescales, the nuclear count rate varied by $\lesssim 2 \sigma(20 \%)$ during the $52 \mathrm{ks}$ observation. We then searched for variability on any scale using an application of the Gregory-Loredo algorithm (1992) on the unbinned events time series. We only found possible variability in the lowest order $m=2$ moment $[\log ($ odds ratio $)=36.9$, with the normalized odds ratio of 0.95$]$, consistent with a slow $(\$ 2 \sigma)$ increase in the mean signal over the duration of this observation. The GregoryLoredo analysis failed to find any statistically significant evidence for variability on shorter timescales, i.e., in the $m>2$ moments. Thus, we find no statistical evidence in these data to support the claim by Xu et al. (2005) that was based on their "visual inspection of the light curve," for X-ray variability on $1 \mathrm{hr}$ timescales.

\section{CONCLUSIONS}

In this work we analyzed a $54.4 \mathrm{ks}$ Chandra observation of the elliptical galaxy NGC 4552 in the Virgo Cluster and found X-ray evidence for shocks in the inner region of the galaxy from a recent nuclear outburst. Two ringlike emission features, consistent with bipolar nuclear outflow cavities, are found in the X-ray images at $r \sim 1.3 \mathrm{kpc}$ from NGC 4552's center. The emission measure weighted gas temperature through the rings $\left(k T_{\text {proj }} \sim\right.$ $0.61 \pm 0.02 \mathrm{keV})$ and the shape of the surface brightness profile across the rim of the northern ring are consistent with a simple spherical model for a Mach 1.7 shock from a $1.4 \times 10^{55}$ ergs nuclear outburst $\sim 1-2$ Myr ago. The mechanical power carried in the shock, $L_{\text {shock }} \sim 3.3 \times 10^{41} \mathrm{ergs} \mathrm{s}^{-1}$, is consistent with that expected from the $L_{\text {mech }}-L_{\text {radio }}$ correlation (Birzan et al. 2004) between the mechanical luminosity and total radio power for $\mathrm{X}$-ray sources showing nuclear outflow activity. One outburst of this magnitude every $\approx 18$ Myr could balance the radiative cooling of NGC 4552's hot ISM.

The X-ray spectrum for the 1".3 circular region containing the nuclear point source is well fit by a two-component power-law + APEC model, with a power-law photon index $\Gamma=1.7 \pm 0.1$ and gas temperature $k T=1.04_{-0.17}^{+0.21}$, in agreement with previous work by Filho et al. (2004). The higher temperature found for gas in the nuclear region compared to that elsewhere in the galaxy suggests that we may be directly observing the reheating of the galaxy ISM in this region by the nuclear outbursts. 
This work is supported in part by NASA grant GO3-4176A and the Smithsonian Institution. This work has made use of the NASA/IPAC Extragalactic Database (NED), which is operated by the Jet Propulsion Laboratory, California Institute of Tech- nology, under contract with the National Aeronautics and Space Administration. We wish to thank Maxim Markevitch for the use of his edge analysis codes and Arnold Rots for help in analyzing the nuclear point source variability.
Bautz, M. W., et al. 1998, Proc. SPIE, 3444, 210

Best, P. N., Kaiser, C. R., Heckman, T. M., \& Kauffmann, G. 2006, MNRAS, 368, L67

Binney, J., \& Tabor, G. 1995, MNRAS, 276, 663

Birzan, L., Rafferty, D. A., McNamara, B. R., Wise, M. W., \& Nulsen, P. E. J. 2004, ApJ, 607, 800

Brüggen, M., \& Kaiser, C. R. 2002, Nature, 418, 301

Cappellari, M., Renzini, A., Greggio, L., di Serego Alighieri, S., Buson, L. M., Burstein, D., \& Bertola, F. 1999, ApJ, 519, 117

Churazov, E., Brüggen, M., Kaiser, C. R., Böhringer, H., \& Forman, W. R. 2001, ApJ, 554, 261

Croton, D., Springel, V., White, S. D. M., De Lucia, G., Frenk, C. S., Gao, L., Jenkins, A, Kauffmann, G., Navarro, J. F., \& Yoshida, Y. 2006, MNRAS, 365,11

Dickey, J. M., \& Lockman, F. J. 1990, ARA\&A, 28, 215

Fabian, A. 1994, ARA\&A, 32, 277

Fabian, A., Reynolds, C., Taylor, G., \& Dunn, R. 2005, MNRAS, 363, 891

Fabian, A. C., Sanders, J. S., Allen, S. W., Crawford, C. S., Iwasawa, K., Johnstone, R. M., Schmidt, R. W., \& Taylor, G. B. 2003, MNRAS, 344, L43

Ferrarese, L., \& Merritt, D. 2000, ApJ, 539, L9

Filho, M. E., Barthel, P. D., \& Ho, L.C 2000, ApJS, 129, 93

Filho, M. E., Fraternali, F., Markoff, S., Nagar, N. M., Barthel, P. D., Ho, L. D., \& Yuan, F. 2004, A\&A, 418, 429

Forman, W., et al. 2005, ApJ, 635, 894

Garmire, G. P., et al. 1992, AIAA Space Programs and Technologies Conference (Paper 92-1473; New York: AIAA)

Gebhardt, K., et al. 2000, ApJ, 539, L13

Gilfanov, M. 2004, MNRAS, 349, 146

Granato, G.L, De Zotti, G., Silva, L., \& Bressan, A. 2004, ApJ, 600, 580

Gregory, P. C., \& Loredo, T. J. 1992, ApJ, 398, 146

Heinz, S., \& Churazov, E. 2005, ApJ, 634, L141

Heinz, S., Reynolds, C, \& Begelman, M. 1998, ApJ, 501, 126

Helou, G., Khan, I., Malek, L., \& Boehmer, L. 1988, ApJS, 68, 151

Ho, L. C., Filippenko, A. V., \& Sargent, W. L. W. 1997, ApJS, 112, 315

Kennicutt, R. C., Jr. 1998, ARA\&A, 36, 189

Landau, L. D., \& Lifschitz, E. M. 1959, Fluid Mechanics (London: Pergamon), chapter 9

Machacek, M., Jones, C., Forman, W. R., \& Nulsen, P. E. J. 2006, ApJ, 644, 155

\section{EFERENCES}

Maoz, D., Waxman, E., \& Loeb, A. 2005, ApJ, 625, 699

Markevitch, M., et al. 2000, ApJ, 541, 542

Matsushita, K. 2001, ApJ, 547, 693

McNamara, B., Wise, M., \& Murry, S. S. 2004, ApJ, 601, 173

McNamara, B., et al. 2000, ApJ, 534, L135

Morrison, R., \& McCammon, D. 1983, ApJ, 270, 119

Nagar, N. M., Falcke, H., Wilson, A. S., \& Ulvestad, J. S. 2002, A\&A, 392, 53

Nulsen, P. E. J., Hambrick, D. C., McNamara, B. R., Rafferty, D., Birzan, L., Wise, M. W., \& David, L. P. 2005a, ApJ, 625, L9

Nulsen, P. E. J., McNamara, B. R., Wise, M. W., \& David, L. P. 2005b, ApJ, 628,629

Omma, H., Binney, J., Bryan, G., \& Slyz, A. 2004, MNRAS, 348, 1105

Owen, F., Eilek, J., \& Kassim, N. 2000, ApJ, 543, 611

Peterson, J., Kahn, J, Paerels, F., Kaastra, J., Tamura, T., Bleeker, J., Ferrigno, C., \& Jernigan, J. 2003, ApJ, 590, 207

Plucinsky, P. P., et al. 2003, Proc. SPIE, 4851, 89

Quilis, V., Bower, R. G.,\& Balogh, M. L. 2001, MNRAS, 328, 1091

Renzini, A., Greggio, L., di Serego Allghieri, S., Capellari, M., Burstein, D., \& Bertola, F. 1995, Nature, 378, 39

Reynolds, C., Heinz, S., \& Begelman, M. 2001, ApJ, 549, L179

Reynolds, C., McKernan, B., Fabian, A., Stone, J., \& Vernaleo, J. 2005, MNRAS, 357, 242

Ruszkowski, M., Brüggen, M., \& Begelman, M. 2004, ApJ, 615, 675

Schindler, S., Binggeli, B., \& Böhringer, H. 1999, A\&A, 343, 420

Silk, J., Djorgovski, S., Wyse, R., \& Bruzual, C. 1986, ApJ, 307, 415

Smith, R. K., Brickhouse, N. S., Liedahl, D. A., \& Raymond, J. D. 2001, ApJ, 556, L91

Tamura, T., et al. 2001, A\&A, 365, 87

Terashima, Y., \& Wilson, A. S. 2003, ApJ, 583, 145

Terlevich, A. I., \& Forbes, D. A. 2002, MNRAS, 330, 547

Tonry, J. L., Dressler, A., Blakeslee, J. P., Ajhar, E. A., Fletcher, A. B., Luppino,

G. A. Metzger, M. R., \& Moore, C. B. 2001, ApJ, 546, 681

van Dokkum, P. G., \& Franx, M. 1995, AJ, 110, 2027

Vikhlinin, A., Markevitch, M., \& Murray, S. S. 2001, ApJ, 551, 160

Wise, M, O'Connell, R., Bregman, J., \& Roberts, M. 1993, ApJ, 405, 94

Xu, Y., Xu, H., Zhang, Z., Kundu, A., Wang, Y., \& Wu, X.-P. 2005, ApJ, 631, 809

Young, A., Wilson, A., \& Mandell, C. 2002, ApJ, 579, 560 